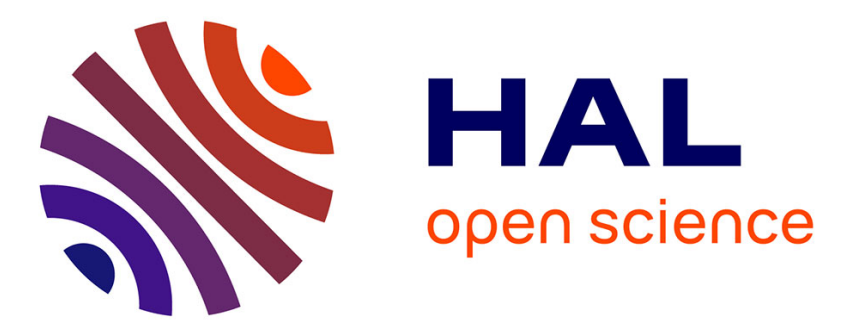

\title{
Astrometric and Light-Travel Time Orbits to Detect Low-Mass Companions: A Case Study of the Eclipsing System R Canis Majoris
}

Ignasi Ribas, Frédéric Arenou, Edward F Guinan

\section{- To cite this version:}

Ignasi Ribas, Frédéric Arenou, Edward F Guinan. Astrometric and Light-Travel Time Orbits to Detect Low-Mass Companions: A Case Study of the Eclipsing System R Canis Majoris. The Astronomical Journal, 2002, 123 (4), pp.2033-2041. 10.1086/339560 . hal-02054276

\section{HAL Id: hal-02054276 https://hal.science/hal-02054276}

Submitted on 1 Mar 2019

HAL is a multi-disciplinary open access archive for the deposit and dissemination of scientific research documents, whether they are published or not. The documents may come from teaching and research institutions in France or abroad, or from public or private research centers.
L'archive ouverte pluridisciplinaire HAL, est destinée au dépôt et à la diffusion de documents scientifiques de niveau recherche, publiés ou non, émanant des établissements d'enseignement et de recherche français ou étrangers, des laboratoires publics ou privés. 


\title{
ASTROMETRIC AND LIGHT-TRAVEL TIME ORBITS TO DETECT LOW-MASS COMPANIONS: A CASE STUDY OF THE ECLIPSING SYSTEM R CANIS MAJORIS
}

\author{
Ignasi Ribas, ${ }^{1,2}$ FrédÉRIC ARENOU, ${ }^{3}$ AND EdWARd F. GUINAN ${ }^{1}$ \\ Received 2001 December 3; accepted 2002 January 8
}

\begin{abstract}
We discuss a method to determine orbital properties and masses of low-mass bodies orbiting eclipsing binaries. The analysis combines long-term eclipse timing modulations (the light-travel time [LTT] effect) with short-term, high-accuracy astrometry. As an illustration of the method, the results of a comprehensive study of Hipparcos astrometry and over 100 years of eclipse timings of the Algol-type eclipsing binary R Canis Majoris are presented. A simultaneous solution of the astrometry and the LTTs yields an orbital period of $P_{12}=92.8 \pm 1.3 \mathrm{yr}$, an LTT semiamplitude of $2574 \pm 57 \mathrm{~s}$, an angular semimajor axis of $a_{12}=117 \pm 5$ mas, and values of the orbital eccentricity and inclination of $e_{12}=0.49 \pm 0.05$ and $i_{12}=91.7 \pm 4.7$, respectively. Adopting the total mass of R CMa of $M_{12}=1.24 \pm 0.05 M_{\odot}$, the mass of the third body is $M_{3}=0.34 \pm 0.02$ $M_{\odot}$, and the semimajor axis of its orbit is $a_{3}=18.7 \pm 1.7$ AU. From its mass, the third body is either a dM34 star or, more unlikely, a white dwarf. With the upcoming microarcsecond-level astrometric missions, the technique that we discuss can be successfully applied to detect and characterize long-period planetary-size objects and brown dwarfs around eclipsing binaries. Possibilities for extending the method to pulsating variables or stars with transiting planets are briefly addressed.
\end{abstract}

Key words: astrometry — binaries: eclipsing — stars: fundamental parameters stars: individual (R Canis Majoris) — stars: late-type

\section{INTRODUCTION}

Within the next decade several space astrometry missions, the Full-Sky Astrometric Mapping Explorer (FAME) and the Double Interferometer for Visual Astrometry (DIVA) and then the Space Interferometry Mission (SIM) and $G A I A$, capable of submilliarcsecond to microarcsecond accuracy are expected to be launched. One of the primary scientific goals of these missions is the astrometric detection of low-mass objects around nearby stars, including brown dwarfs and Jupiter-sized planets. The detection of these objects will be accomplished through the observation of the reflex motion of the host star caused by the gravitational pull of the low-mass body. Although these missions are capable of very high astrometric accuracies and can observe up to millions of stars, their lifetimes are relatively short (2.5-5 yr). Thus, these space missions are optimized to detect planets within the habitable zones of late-type stars, but they could fail to detect (additional) planets with longer periods. It is important, however, to secure a complete picture of the bodies orbiting a star both from a pure census point of view and also to understand the genesis and evolution of planetary systems. In addition, planets do not necessarily remain within the habitable zone because of longterm chaotic perturbations. As we know from our solar system, the presence of massive planets, such as Jupiter and Saturn, in distant orbits plays a crucial role in stabilizing the orbits of the inner planets.

\footnotetext{
${ }^{1}$ Department of Astronomy and Astrophysics, Villanova University, Villanova, PA 19085; iribas@ast.villanova.edu, edward.guinan@ villanova.edu.

${ }^{2}$ Departament d'Astronomia i Meteorologia, Universitat de Barcelona, Avenida Diagonal 647, E-08028 Barcelona, Spain.

${ }^{3}$ DASGAL, Observatoire de Paris, CNRS UMR 8633, F92195 Meudon Cedex, France; frederic.arenou@obspm.fr.
}

One effective way of extending the time baseline that permits the discovery of long-period exosolar planets or brown dwarfs is to use the light-travel time (LTT) effect in eclipsing binaries. From this technique, the eclipses act as an accurate clock for detecting subtle variations in the distance to the object (this is analogous to the method used for discovering Earth-sized objects around pulsars; see Wolszczan \& Frail 1992). The periodic quasi-sinusoidal variations of the eclipse arrival times have a very simple and direct physical meaning: the total path that the light has to travel varies periodically as the eclipsing pair moves around the barycenter of the triple system. The amplitude of the variation is proportional to both the mass and the period of the third body as well as to the sine of the orbital inclination. As discussed by Demircan (2000), nearly 60 eclipsing binaries show evidence for nearby, unseen tertiary components using LTT effects. A recent example of a brown dwarf detected around the eclipsing binary V471 Tau using this method was presented by Guinan \& Ribas (2001). Also, this method is being employed in selected low-mass eclipsing binaries to search for extrasolar planets (Deeg et al. 2000).

The primary advantages of using the LTT effect to detect third bodies in eclipsing binaries are that (1) the necessary photometry can be secured with small telescopes using photoelectric or CCD detectors, (2) the number of eclipsing binaries is large — 4000 currently known in the Galaxy — and this number could increase very significantly when results from upcoming astrometry and photometry missions (e.g., MONS, COROT, Eddington, Kepler) are available, (3) for select eclipsing binaries (with sharp and deep eclipses), the timings can be determined with accuracies as good as several seconds, and (4) the mass of the eclipsing pair can be known from conventional spectroscopic and light-curve analyses. A shortcoming of the LTT method is that only upper limits to the mass and size of the orbit of the tertiary component can be determined [the analysis yields the mass 
function ${ }^{4} f\left(M_{3}\right)$ and $a_{3} \sin i_{3}$. However, as was demonstrated in the case of Algol (Bachmann \& Hershey 1975), the LTT analysis can be complemented with astrometry to yield the orbital inclination and thus the actual mass and semimajor axis of the third body. Furthermore, with the orbital properties $(P, e$, and $\omega)$ known from the LTT analysis only a small fraction of the astrometric orbit needs to be covered when using high-accuracy astrometry.

In this paper we present the results of the combined LTT analysis and Hipparcos astrometry of the Algol-type eclipsing binary $\mathrm{R}$ Canis Majoris. The residuals of over 150 eclipse timings extending from 1887 to 2001 show a periodic ( $93 \mathrm{yr}$ ) quasi-sinusoidal modulation. As previously shown by Radhakrishnan, Sarma, \& Abhyankar (1984) and Demircan (2000), these variations are best explained by the LTT effect arising from the gravitational influence of a third body. The Hipparcos astrometry also shows the presence of small but significant acceleration terms in the propermotion components explicable by the reflex motion from a third body. Our study illustrates that with a well-defined LTT effect, only a few years of accurate astrometry are needed to constrain the orbital solution and determine the mass of the third body.

\section{OVERVIEW OF R CMa}

$\mathrm{R}$ Canis Majoris (HD 57167, HR 2788, HIP 35487) is a bright $\left(V_{\max }=5.67 \mathrm{mag}\right)$, semidetached eclipsing binary having an orbital period of 1.1359 days. As pointed out by Varricatt \& Ashok (1999), R CMa holds special status among Algol systems in that it is the system with the lowest known total mass and hosting the least massive secondary star. Since the discovery of its variability in 1887 by Sawyer (1887), R CMa has been frequently observed and has welldetermined orbital and physical properties. The major breakthrough in understanding the system came when Tomkin (1985) was able to measure the very weak absorption lines of the faint secondary star and determine the masses of the two stars from a double-line radial velocity study. The analyses of its light and radial velocity curves (see Varricatt \& Ashok 1999) show that this system has a circular orbit and consists of a nearly spherical F0-1 V star $\left(M_{1}=1.07 \pm 0.2 \quad M_{\odot}, \quad R_{1}=1.48 \pm 0.10 \quad R_{\odot}, \quad L_{1} / L_{\odot}=\right.$ $5.78 \pm 0.38)$ and a low-mass, tidally distorted K2-3 IV star $\left(M_{2}=0.17 \pm 0.02 \quad M_{\odot}, \quad R_{2}=1.06 \pm 0.07 \quad R_{\odot}, \quad L_{2} / L_{\odot}=\right.$ $0.43 \pm 0.10$ ). Moreover, nearly every photometric study indicates that the cooler star fills its inner Lagrangian surface. The relatively high space motions $\left(S=67 \mathrm{~km} \mathrm{~s}^{-1}\right)$ suggest that $\mathrm{R} \mathrm{CMa}$ is a member of the old disk population and thus a fairly old (5-7 Gyr) star (Guinan \& Ianna 1983).

The present state of the system is best explained as a lowmass Algol system that has undergone mass exchange and extensive mass loss. Asymmetries in its light curves and subtle spectroscopic anomalies indicate that mass exchange and loss are still continuing but at a much diminished rate compared to most Algol systems. The very low mass of the secondary star and old disk age indicate that $\mathrm{R} \mathrm{CMa}$ is near the end of its life as an Algol system. As in the case of all Algol systems, the secondary star lies well above the main sequence. However, unlike most Algol systems, the primary

\footnotetext{
${ }^{4} \operatorname{Here} f\left(M_{3}\right)=\left(M_{3}^{3} \sin i_{3}^{3}\right) /\left(M_{12}+M_{3}\right)^{2}$.
}

star is too hot and overluminous for observed mass. Moreover, a recent analysis of older photometry of R CMa by Mkrtichian \& Gamarova (2000) indicates that the F star is a low-amplitude $\delta$ Scuti variable with a B light amplitude of 9 mmag and a period of 68 minutes.

\section{OBSERVATIONS}

\subsection{Astrometry}

Hipparcos observed R CMa between 1990 March 9 and 1993 March 5. There are 68 one-dimensional astrometric measurements corresponding to 35 different epochs in the Hipparcos intermediate astrometric data, which were obtained by the two Hipparcos Data Reduction Consortia (33 measurements from FAST and 35 from the NDAC). The astrometric data can be obtained from CD-ROM 5 of the Hipparcos catalog (Perryman 1997). Unfortunately, the time span of the Hipparcos observations is much smaller than the orbital period of the tertiary component, and this might eventually result in possible systematic errors in the orbital elements. To further constrain the solution, additional older ground-based positions must be used. Indeed, Tycho-2 proper motions were computed by combining Tycho-2 positions and ground-based astrometric catalogs. For R CMa, 17 epoch positions of ground-based catalogs used for the Tycho-2 proper-motion computation were kindly made available to us by S. E. Urban and are listed in Table 1. These measurements span over one century, and so the Tycho-2 proper motions can be understood as the combination of the true proper motion and a large fraction of the orbital motion. Consequently, the Tycho-2 proper motion of R CMa cannot itself be used in our analysis, and only the individual positions contain valuable orbital information. In contrast, a short-term proper-motion determination, such as the one computed around 1980 by Guinan \& Ianna (1983), reveals itself to be very useful in constraining the astrometric solution.

In the course of the astrometric data reduction of the Hipparcos data, a test was applied to all the (apparently) single stars in order to check whether their motion was significantly nonlinear. Most likely, a significant curvature of the photocenter motion is an indication of a possible duplicity. As it turns out, R CMa is one of the 2622 "acceleration", solutions of the double and multiple stars annex of the Hipparcos Catalogue, which provides a hint for the presence of a third body, independently of the LTT effect.

\subsection{Photometry}

R CMa has a long baseline of eclipse timings that extend from the present back to 1887 . Most of the early eclipse times were determined from visual estimates. Several period studies have been carried out. Early studies of times of minimum light indicated a possible abrupt decrease in the period during 1914-1915 (see Dugan \& Wright 1939; Wood 1946; Koch 1960; Guinan 1977). However, as more timings accumulated, it became apparent that the long-term variations in the $(O-C)$ values of the system are periodic and thus best explained by the LTT effect produced by the presence of a third body. The analyses of Radhakrishnan et al. (1984), with eclipse timings from 1887 to 1982, and Demircan (2000), who includes timings up to 1998, make a strong case in support of the LTT scenario. 
TABLE 1

Ground-Based Astrometric Data For R CMA

\begin{tabular}{|c|c|c|c|c|c|}
\hline Julian Year & $\begin{array}{c}\alpha \\
\text { (deg) }\end{array}$ & $\begin{array}{c}\delta \\
(\mathrm{deg})\end{array}$ & $\begin{array}{c}\sigma_{\alpha} \cos \delta \\
\text { (mas) }\end{array}$ & $\begin{array}{c}\sigma_{\delta} \\
\text { (mas) }\end{array}$ & Source \\
\hline $1894.7 \ldots \ldots \ldots \ldots$ & 109.86244875 & -16.391100556 & 861 & 690 & WASH AG 1900 \\
\hline $1905.15 \ldots \ldots \ldots$ & 109.8630725 & -16.391588056 & 253 & 269 & A.C. \\
\hline $1914.11 \ldots \ldots .$. & 109.863289583 & -16.391985556 & 132 & 116 & CAPE 2ND FUND 1900 \\
\hline $1914.97 \ldots \ldots .$. & 109.863497083 & -16.391931111 & 304 & 332 & A.C. \\
\hline $1916.2 \ldots \ldots \ldots .$. & 109.863377917 & -16.392131667 & 294 & 282 & ALBANY 10 \\
\hline $1918.5 \ldots \ldots \ldots . .$. & 109.863659583 & -16.392145833 & 321 & 270 & WASH 20 \\
\hline $1923.05 \ldots \ldots .$. & 109.863762083 & -16.392368333 & 156 & 153 & CAPE $1-25$ \\
\hline $1933.52 \ldots \ldots \ldots$ & 109.864227083 & -16.392731944 & 316 & 264 & YALE $12 / 1-14 /-18$ \\
\hline $1934.44 \ldots \ldots \ldots$ & 109.86428875 & -16.392647222 & 237 & 210 & CAPE 3-25 \\
\hline $1939.58 \ldots \ldots \ldots$ & 109.86449 & -16.392880556 & 130 & 132 & CAPE $1-50$ \\
\hline $1942.02 \ldots \ldots .$. & 109.864635833 & -16.393026944 & 151 & 200 & WASH 40 9-IN \\
\hline $1969.33 \ldots \ldots \ldots$ & 109.865958333 & -16.394058889 & 45 & 45 & $\mathrm{CPC} 2$ \\
\hline $71983.05 \ldots \ldots$. & 109.866597083 & -16.394559722 & 168 & 164 & WASH TAC \\
\hline $1984.37 \ldots \ldots \ldots$ & 109.866713333 & -16.394581389 & 86 & 181 & PERTH 83 \\
\hline $1986 \ldots \ldots \ldots \ldots$ & 109.8667525 & -16.394728333 & 88 & 119 & CAMC Series \\
\hline $1986.07 \ldots \ldots .$. & 109.866875417 & -16.394721944 & 110 & 119 & FOKAT \\
\hline $1992.49 \ldots \ldots .$. & 109.8670625 & -16.394969444 & 39 & 46 & WASH 2-J00 \\
\hline
\end{tabular}

Note.-References for sources: WASH: S. E. Urban 2001, private communication; A.C.: Urban et al. 1998; CAPE: S. E. Urban 2001; private communication; ALBANY: S. E. Urban 2001; private communication; YALE: S. E. Urban 2001, private communication; CPC2: Zacharias et al. 1992; PERTH: S. E. Urban 2001, private communication; CAMC: Fabricius 1993; FOKAT: Bystrov et al. 1991.

Our photoelectric eclipse timing observations extend the time baseline up to early 2001. The observations were obtained with the Four College $0.8 \mathrm{~m}$ Automatic Photoelectric Telescope located in southern Arizona during 1995/ 1996 and 2000/2001. Differential photometry was carried out using $u v b y$ Strömgren filter sets. The midtimes of primary minimum and the $(O-C)$ values for these are given in Table 2, along with the corresponding uncertainties. The $(O-C)$ values were computed using a refined ephemeris determined from the analysis in $\S 4$ (eq. [5]). Our observations were combined with those compiled from the literature to yield a total of 158 eclipse timings from 1887 through 2001. The primary-eclipse observations obtained from the literature are also provided in Table 2. Even though it is not explicitly mentioned in any of the publications, the times listed are commonly assumed to be in the coordinated universal timescale (UTC). The timings were transformed from UTC to terrestrial time (TT) following the procedure described in Guinan \& Ribas (2001), which is based on the recommendations of Bastian (2000). The timings listed in Table 2 are therefore HJD but in the TT scale.

The uncertainties of the individual timings are difficult to estimate. The compilation of Radhakrishnan et al. (1984; from which most of the timings in Table 2 come) does not provide timing errors but only a relative weighting factor related to the quality of the data and the observation technique. We therefore adopted an iterative scheme to determine the actual uncertainties by forcing the $\chi^{2}$ of the $(O-C)$ curve fit (in $\S 4$ ) to be equal to unity. This rather arbitrary scale factor determination is indeed justified because it ensures that the fitting algorithm will yield realistic uncertainties for the orbital parameters of the system. The individual timing errors are included in Table 2 . The uncertainties yielded by the iterative scheme are about 10-13 minutes for photographic timings and some 2-4 minutes for photoelectric timings, in both cases reasonable figures given the characteristics of the two methods and the shape of the eclipse.

The Hipparcos mission, in addition to observing accurate positions of $\mathrm{R} \mathrm{CMa}$, obtained a total of 123 photometric measurements, which are present in the Hipparcos epoch photometric data (Perryman 1997). The phase coverage of the observations is not sufficient to determine an accurate primary-eclipse timing using conventional methods. As an alternative, we adopted the physical information available to fit the entire light curve and derive a phase offset. Also, since the observations span $3 \mathrm{yr}$ and the system exhibits $(O-C)$ variations, the photometric data set was split into two subgroups about 1991 January 1. Then, using the photometric elements of Varricatt \& Ashok (1999), we employed the Wilson-Devinney program (Wilson \& Devinney 1971) to run fits to both light curves by leaving only a phase shift and a magnitude zero point as free parameters. The fits were very satisfactory, and we derived eclipse timings for the mean epochs from the best-fitting phase offsets. The resulting two timings, with uncertainties of around 100 $\mathrm{s}$, are included in Table 2.

These two timings are significantly different, as one would expect from the LTT secular change of period during the 3 yr duration of the Hipparcos observations. More precisely, each date of observation could be approximated by $T \approx T_{0}+P_{0} E+g E^{2}$, with $E$ being the variability cycle number. So, the photometric "acceleration" term $g$ is a measure of the departure from a linear ephemeris during the observation window (the Hipparcos mission lifetime in this case). Since Hipparcos did not provide minimum timings, we used an alternative method to fit the equation above. The epoch measurements were folded using the reference epoch and period from ground-based studies. Then the quadratic term was estimated by minimizing the distance between successive points of the folded light curve (stringlength method). The fit yielded a value of $g=2.09 \times 10^{-8}$ 
TABLE 2

Primary-Eclipse Timings For R CMA

\begin{tabular}{|c|c|c|c|c|c|c|c|c|c|c|c|}
\hline $\mathrm{HJD}^{\mathrm{a}}$ & $\begin{array}{c}(\mathrm{O}-\mathrm{C}) \\
(\mathrm{s})\end{array}$ & $\begin{array}{l}\sigma^{\mathrm{b}} \\
(\mathrm{s})\end{array}$ & Reference & $\mathrm{HJD}^{\mathrm{a}}$ & $\begin{array}{c}(O-C) \\
(\mathrm{s})\end{array}$ & $\begin{array}{l}\sigma^{\mathrm{b}} \\
\text { (s) }\end{array}$ & Reference & $\mathrm{HJD}^{\mathrm{a}}$ & $\begin{array}{c}(O-C) \\
(\mathrm{s})\end{array}$ & $\begin{array}{l}\sigma^{\mathrm{b}} \\
(\mathrm{s})\end{array}$ & Reference \\
\hline $2410368.9939 \ldots$ & -3114 & 610 & 1 & $2436982.9957 \ldots$ & -1608 & 240 & 1 & $2442402.5785 \ldots$ & -1287 & 770 & 1 \\
\hline $2410562.1139 \ldots$. & -2262 & 610 & 1 & $2437378.3104 \ldots$ & -1012 & 770 & 4 & $2442426.4195 \ldots$ & -2477 & 770 & 1 \\
\hline $2410664.3469 \ldots$ & -2415 & 610 & 1 & $2437696.3624 \ldots$ & -2027 & 770 & 4 & $2442426.4225 \ldots$. & -2218 & 770 & 1 \\
\hline $2411425.4369 \ldots$ & -1648 & 610 & 1 & $2437746.3434 \ldots$ & -2065 & 770 & 4 & $2442467.3005 \ldots$ & -3593 & 770 & 1 \\
\hline $2411993.3909 \ldots$. & -3116 & 610 & 1 & $2438089.4114 \ldots$ & -896 & 770 & 4 & $2442785.3675 \ldots$ & -3311 & 770 & 1 \\
\hline $2412527.3029 \ldots$ & -1451 & 770 & 1 & $2438105.3104 \ldots$ & -1258 & 610 & 4 & $2442802.4325 \ldots$ & -1076 & 770 & 1 \\
\hline $2413242.9557 \ldots$ & -642 & 610 & 1 & $2438114.3994 \ldots$ & -1132 & 770 & 4 & $2442802.4345 \ldots$ & -903 & 770 & 1 \\
\hline $2414333.4539 \ldots$ & -1166 & 610 & 1 & $2438384.7384 \ldots$ & -2443 & 610 & 4 & $2442820.5941 \ldots$ & -2240 & 240 & 1 \\
\hline $2414447.0559 \ldots$ & -491 & 400 & 1 & $2438399.5192 \ldots$ & -1272 & 520 & 4 & $2442826.2775 \ldots$ & -1921 & 770 & 1 \\
\hline $2414878.7180 \ldots$ & -140 & 610 & 1 & $2438400.6454 \ldots$ & -2114 & 610 & 4 & $2442826.2865 \ldots$ & -1143 & 770 & 1 \\
\hline $2415810.2070 \ldots$. & 1296 & 450 & 1 & $2438406.3387 \ldots$ & -940 & 520 & 4 & $2442835.3485 \ldots$ & -3350 & 770 & 1 \\
\hline $2416718.9560 \ldots$. & 904 & 610 & 2 & $2438440.4174 \ldots$ & -902 & 770 & 4 & $2442835.3685 \ldots$ & -1622 & 770 & 1 \\
\hline $2418309.2921 \ldots$ & 2400 & 610 & 1 & $2438817.5334 \ldots$ & -2347 & 770 & 4 & $2443161.3775 \ldots$ & -2169 & 770 & 1 \\
\hline $2419615.6312 \ldots$. & 2901 & 770 & 1 & $2438818.6687 \ldots$ & -2402 & 290 & 4 & $2443162.5145 \ldots$ & -2077 & 770 & 1 \\
\hline $2419849.6342 \ldots$. & 2811 & 450 & 1 & $2438832.3054 \ldots$ & -1936 & 770 & 4 & $2443186.3616 \ldots$ & -2750 & 770 & 1 \\
\hline $2420138.1572 \ldots$. & 2271 & 770 & 1 & $2439140.1446 \ldots$ & -2028 & 240 & 1 & $2443202.2725 \ldots$ & -2075 & 770 & 1 \\
\hline $2420513.0292 \ldots$. & 3236 & 770 & 1 & $2439164.0002 \ldots$ & -1957 & 240 & 1 & $2443203.3966 \ldots$ & -3106 & 770 & 1 \\
\hline $2421278.6462 \ldots$. & 2557 & 450 & 1 & $2439169.6784 \ldots$ & -2087 & 770 & 1 & $2443219.3125 \ldots$ & -1999 & 770 & 1 \\
\hline $2421648.9832 \ldots$. & 4279 & 770 & 1 & $2439492.2922 \ldots$ & -1544 & 610 & 5 & $2443430.5776 \ldots$ & -3744 & 770 & 1 \\
\hline $2422029.5022 \ldots$. & 2417 & 450 & 1 & $2439518.4104 \ldots$. & -2275 & 520 & 4 & $2443512.3796 \ldots$ & -2519 & 770 & 1 \\
\hline $2422030.6382 \ldots$. & 2422 & 400 & 1 & $2439528.6364 \ldots$ & -2057 & 770 & 4 & $2443513.5136 \ldots$ & -2687 & 770 & 1 \\
\hline $2422558.8492 \ldots$. & 2249 & 610 & 1 & $2439533.1794 \ldots$ & -2124 & 240 & 1 & $2443587.3586 \ldots$ & -1929 & 770 & 1 \\
\hline $2422765.5903 \ldots$. & 2211 & 450 & 1 & $2439802.4034 \ldots$. & -1626 & 240 & 1 & $2443595.2966 \ldots$ & -3103 & 770 & 1 \\
\hline $2423098.4213 \ldots$. & 2212 & 610 & 1 & $2439822.8464 \ldots$ & -1967 & 770 & 1 & $2443612.3376 \ldots$ & -2942 & 770 & 1 \\
\hline $2423406.2533 \ldots$. & 1498 & 520 & 1 & $2439863.7384 \ldots$ & -2132 & 770 & 1 & $2443880.4246 \ldots$ & -2535 & 770 & 1 \\
\hline $2423442.6093 \ldots$. & 2004 & 400 & 1 & $2439870.5314 \ldots$ & -4090 & 770 & 1 & $2443888.3706 \ldots$ & -3018 & 770 & 1 \\
\hline $24238666.3213 \ldots$. & 2492 & 770 & 1 & $2439872.8174 \ldots$ & -2870 & 770 & 1 & $2443905.4166 \ldots$ & -2425 & 770 & 1 \\
\hline $2424667.1393 \ldots$. & 671 & 610 & 1 & $2439875.0984 \ldots$ & -2082 & 80 & 1 & $2443946.3066 \ldots$. & -2763 & 770 & 1 \\
\hline $2425052.2353 \ldots$. & 1679 & 610 & 1 & $2439896.6704 \ldots$ & -3024 & 770 & 1 & $2443971.2946 \ldots$ & -2998 & 770 & 1 \\
\hline $2425320.3193 \ldots$. & 1825 & 450 & 1 & $2439904.6324 \ldots$. & -2125 & 770 & 1 & $2444255.2839 \ldots$ & -2668 & 240 & 1 \\
\hline $2425650.8783 \ldots$. & 1816 & 770 & 1 & $2439905.7724 \ldots$ & -1774 & 770 & 1 & $2444281.4036 \ldots$ & -3270 & 770 & 1 \\
\hline $2425990.5203 \ldots$. & 1415 & 770 & 1 & $2439912.5774 \ldots$ & -2694 & 770 & 1 & $2444606.2986 \ldots$ & -1921 & 240 & 1 \\
\hline $2426014.3803 \ldots$. & 1866 & 610 & 1 & $2439912.5847 \ldots$ & -2064 & 240 & 1 & $2444607.4327 \ldots$ & -2080 & 240 & 1 \\
\hline $2426027.9993 \ldots$. & 803 & 770 & 1 & $2439912.5924 \ldots$ & -1398 & 770 & 1 & $2444647.1938 \ldots$. & -1810 & 240 & 1 \\
\hline $2426753.8563 \ldots$. & -53 & 770 & 1 & $2439929.6374 \ldots$. & -891 & 770 & 1 & $2444648.3289 \ldots$ & -1883 & 240 & 1 \\
\hline $2426994.6883 \ldots$. & 1009 & 610 & 1 & $2439935.3075 \ldots$ & -1730 & 240 & 1 & $2444649.4616 \ldots$ & -2163 & 770 & 1 \\
\hline $2428596.3576 \ldots$. & 242 & 770 & 1 & $2439954.5965 \ldots$ & -3632 & 770 & 1 & $2444664.2304 \ldots$. & -2028 & 240 & 1 \\
\hline $2428922.3748 \ldots$. & 403 & 770 & 1 & $2440288.5785 \ldots$ & -2330 & 770 & 1 & $2444672.1848 \ldots$ & -1786 & 240 & 1 \\
\hline $2429301.7763 \ldots$. & 133 & 240 & 1 & $2440313.5715 \ldots$ & -2133 & 770 & 1 & $2444998.1898 \ldots$ & -2679 & 240 & 1 \\
\hline $2429308.5903 \ldots$. & -8 & 240 & 1 & $2440582.7835 \ldots$ & -2672 & 770 & 1 & $2444999.3295 \ldots$. & -2354 & 240 & 1 \\
\hline $2429309.7273 \ldots$. & 82 & 240 & 1 & $2440591.8781 \ldots$ & -2061 & 240 & 6 & $2445015.2389 \ldots$ & -1817 & 240 & 1 \\
\hline $2429660.7283 \ldots$. & -355 & 450 & 1 & $2440964.4665 \ldots$ & -2109 & 240 & 1 & $2445391.2370 \ldots$ & -1704 & 240 & 7 \\
\hline $2430035.5853 \ldots$. & -687 & 520 & 1 & $2440971.2825 \ldots$ & -2079 & 240 & 1 & $2448137.9592 \ldots$ & -449 & 80 & 8 \\
\hline $2432999.2353 \ldots$. & -2634 & 770 & 1 & $2440979.2345 \ldots$ & -2044 & 240 & 1 & $2448608.2433 \ldots$ & -93 & 80 & 8 \\
\hline $2433367.3203 \ldots$. & 804 & 770 & 1 & $2440995.1395 \ldots$ & -1887 & 240 & 1 & $2450088.3866 \ldots \ldots$ & 850 & 240 & 9 \\
\hline $2434453.2714 \ldots$. & -16 & 770 & 1 & $2440996.2715 \ldots$ & -2228 & 240 & 1 & $2450096.3415 \ldots$ & 1136 & 240 & 9 \\
\hline $2434454.4043 \ldots$. & -270 & 770 & 1 & $2441725.5335 \ldots$ & -3327 & 770 & 1 & $2450107.6995 \ldots$ & 1013 & 240 & 8 \\
\hline $2434481.6620 \ldots$. & -694 & 240 & 1 & $2441765.3075 \ldots$ & -1941 & 770 & 1 & $2450145.1826 \ldots$ & 756 & 240 & 9 \\
\hline $2435515.3604 \ldots$. & -1461 & 240 & 1 & $2442059.5105 \ldots$ & -2456 & 770 & 1 & $2450154.2670 \ldots$ & 485 & 240 & 9 \\
\hline $2435534.6759 \ldots$. & -1074 & 240 & 1 & $2442092.4525 \ldots$ & -2484 & 770 & 1 & $2450439.3955 \ldots$ & 1096 & 240 & 9 \\
\hline $2436958.0042 \ldots$. & -1675 & 240 & 1 & $2442099.2715 \ldots$ & -2194 & 770 & 1 & $2451896.8199 \ldots$ & 2036 & 150 & 8 \\
\hline $2436959.1430 \ldots$. & -1428 & 240 & 1 & $2442100.4005 \ldots$ & -2794 & 770 & 1 & $2451945.6648 \ldots$ & 1977 & 80 & 8 \\
\hline $2436977.3169 \ldots$. & -1530 & 240 & 3 & $2442116.3045 \ldots$ & -2724 & 770 & 1 & & & & \\
\hline
\end{tabular}

a Not in the UTC but in the TT scale (see text).

$\mathrm{b}$ The uncertainties have been computed using the procedure outlined in $\S 3.2$.

References.- (1) Radhakrishnan et al. 1984; (2) Wood 1946; (3) Knipe 1963; (4) Kitamura 1969; (5) Robinson 1967; (6) E. F. Guinan, unpublished; (7) Edalati, Khalesse, \& Riazi 1989; (8) this work; (9) Varricatt \& Ashok 1999.

day, which leads to a cumulative effect of $g E_{\max }^{2}=0.0193 \pm$ 0.0006 day during the course of the Hipparcos observations. This rough estimation gives a significant acceleration term of the same order as that resulting from the long-term LTT analysis $(\S 4)$ and indicates that the $(O-C)$ variations attributed here to the presence of a third body could, in principle, have been detected through the Hipparcos photometric analysis alone. 


\section{ANALYSIS}

The expressions that describe the LTT effect as a function of the orbital properties were first provided by Irwin (1952). In short, the time delay or advance caused by the influence of a tertiary component can be expressed as

$\Delta T=\frac{a_{12} \sin i_{12}}{c}\left[\frac{1-e_{12}^{2}}{1+e_{12} \cos \nu_{12}} \sin \left(\nu_{12}+\omega_{12}\right)+e_{12} \sin \omega_{12}\right]$,

where $c$ is the speed of light and $a_{12}, i_{12}, e_{12}, \omega_{12}$, and $\nu_{12}$ are the semimajor axis, the inclination, the eccentricity, the argument of the periastron, and the true anomaly (function of time) of the orbit of the eclipsing pair around the barycenter, respectively. As is customary, the orbital inclination $i_{12}$ is measured relative to the plane of the sky. The naming convention adopted throughout this paper uses subscripts " 12 " and " 3 " for the orbital parameters of the eclipsing pair and the tertiary component, respectively, around the common barycenter. Obviously, most of the parameters for the eclipsing pair's and the third body's orbits are identical (such as period, eccentricity, and inclination), but we use the subscript " 12 " because the actual measurements are made strictly for the brighter component of the long-period system. Finally, subscript "EB" refers to the close orbit of the eclipsing binary.

The fit of equation (1) to the timing data would provide a good estimation of the a number of orbital and physical parameters of the system (see, e.g., Guinan \& Ribas 2001). However, both the orbital semimajor axis and the mass of the third body would be affected by a factor $\sin i$, which cannot be determined from the LTT analysis alone. When the LTT analysis is combined with astrometric data, all orbital parameters (including $i$ and even $\Omega$ ) can be determined yielding a full description of the system. The availability of Hipparcos intermediate astrometry permits the fitting of the observations using an astrometric model not accounted for in the standard Hipparcos astrometric solution. In the particular case of R CMa we have considered an orbital model that has been convolved with the astrometric motion (parallax and proper motion). The orbital motion produces the following effect on the coordinates:

$$
\begin{aligned}
\Delta x= & a_{12} \frac{1-e_{12}^{2}}{1+e_{12} \cos \nu_{12}}\left[\cos \left(\nu_{12}+\omega_{12}\right) \sin \Omega_{12}\right. \\
& \left.+\sin \left(\nu_{12}+\omega_{12}\right) \cos \Omega_{12} \cos i_{12}\right], \\
\Delta y= & a_{12} \frac{1-e_{12}^{2}}{1+e_{12} \cos \nu_{12}}\left[\cos \left(\nu_{12}+\omega_{12}\right) \cos \Omega_{12}\right. \\
& \left.-\sin \left(\nu_{12}+\omega_{12}\right) \sin \Omega_{12} \cos i_{12}\right] .
\end{aligned}
$$

In addition, since the Hipparcos measurements are unidimensional, the variation of the measured abscissa $v$ on a great circle is

$$
\begin{aligned}
\Delta v= & \frac{\partial v}{\partial \alpha \cos \delta}(\Delta \alpha \cos \delta+\Delta x)+\frac{\partial v}{\partial \delta}(\Delta \delta+\Delta x)+\frac{\partial v}{\partial \varpi} \Delta \varpi \\
& +\frac{\partial v}{\partial \mu_{\alpha} \cos \delta} \Delta \mu_{\alpha} \cos \delta+\frac{\partial v}{\partial \mu_{\delta}} \Delta \mu_{\delta},
\end{aligned}
$$

where the astrometric components are $(\alpha, \delta)$ for the coordinates, $\left(\mu_{\alpha}, \mu_{\delta}\right)$ for the proper motion, and $\varpi$ for the parallax.

In addition to fitting the orbital and astrometric properties of the system, a timing zero point and a correction to the orbital period of the eclipsing pair [which could lead to a linear secular increase or decrease of the $(O-C)$ values] were also considered. The initial values of the period and the reference epoch were adopted from Varricatt \& Ashok (1999).

The full set of observational equations includes those related to the timing residuals (eq. [1]) and those coming from the astrometric measurements (eqs. [2], [3], and [4]). All these equations were combined together, and the 14 unknown parameters (five for the astrometric components $-\alpha, \delta, \mu_{\alpha}, \mu_{\delta}, \varpi-$ seven for the orbital elements- $a_{12}$, $e_{12}, \omega_{12}, i_{12}, P_{12}, T_{12}^{\text {peri }}, \Omega_{12}$ - one for the reference epoch$T_{0 \mathrm{~EB}}-$ and one for the period of the eclipsing system $\left.-P_{\mathrm{EB}}\right)$ were recovered via a weighted least-squares fit as described in, e.g., Arenou (2001) or Halbwachs et al. (2000). Note that the weights of the individual observations were computed as the inverse of the observational uncertainties squared and multiplied by the corresponding correlation factors. The uncertainties adopted are given in Tables 1 and 2 and in the Hipparcos Catalogue CD-ROM 5.

Because of the short time span of the Hipparcos observations, a large uncertainty on the reflex semimajor axis would exist if the Hipparcos astrometric data were used alone. As a first step toward better constraining the solution, we added the epoch proper motion from Guinan \& Ianna (1983) as an external observation (with the two subsequent equations for both components of the proper motion). To do so, the appropriate equation for the first derivatives of the orbital motion was used. As expected, the quality of the solution improved and yielded a semimajor axis of $a_{12}=140 \pm 16$ mas and a tertiary mass of $M_{3}=0.42 \pm 0.05 M_{\odot}$. Yet, a closer inspection of the residuals revealed that this solution was not fully compatible with the ground-based epoch positions mentioned in $\S 3.1$ since a clear trend appeared in declination. For this reason, we decided to include these positions also in the fit, together with the Guinan \& Ianna proper motion and the photometric $(O-C)$ minimum times. In total, the least-squares fit had 262 equations for 14 parameters to determine. A robust fit approach (McArthur, Jefferys, \& McCartney 1994) was used because of the large dispersion of the ground-based astrometric measurements. The resulting goodness of the fit was 0.63 , and graphical representations of the fits to the eclipse timing residuals and Hipparcos intermediate astrometry are shown in Figure 1. The less accurate ground-based astrometric positions (with standard errors of about 200 mas on average) are not represented in the figure for the sake of clarity.

The resulting best-fitting parameters together with their standard deviations are listed in Table 3. The astrometric solution presented supersedes that of the Hipparcos Catalogue because it is based on a sophisticated model that accounts for the orbital motion and considers ground-based astrometry. Also, Table 3 includes the mass and semimajor axis of $\mathrm{R} \mathrm{CMa} \mathrm{C}$ that follow from the adoption of a total mass for the eclipsing system. Finally, our fit also yields new accurate ephemeris for the eclipsing pair:

$$
T(\min . \mathrm{I})=\text { HJED 24, 30436.5807 }+1.13594197 E,
$$

where all times are in the TT scale and the zero epoch refers to the geometric center of the R CMa orbit. Note that the accuracy of the new period we determine (see Table 3 ) is better than $9 \mathrm{~ms}$. We have considered in our analysis a linear ephemeris as that in equation (5). However, Algol systems 


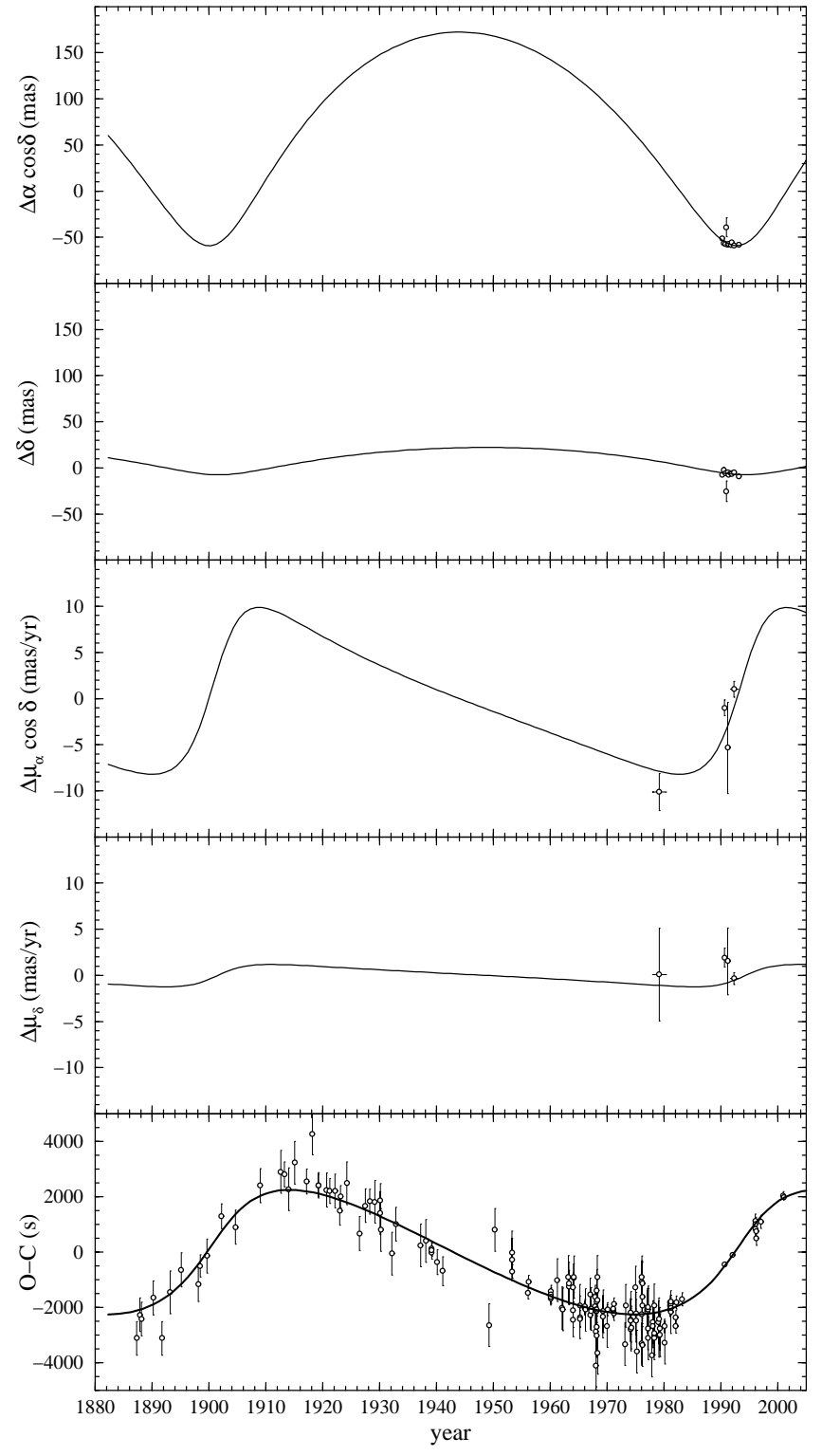

FIG. 1.-Fits to the astrometric data (positions and proper motions in the top four panels and LTT curve in the bottom panel) for R CMa. Note that the Hipparcos data are one-dimensional and thus cannot be represented directly. Instead, we show the Hipparcos position for 10 normal points (epoch groups). Although ground-based positions spanning over one century have been used to constrain the least-square solution, these are not represented for clarity. For the proper motions, the Guinan \& Ianna (1983) estimation and three Hipparcos normal points are represented.

have been observed to experience secular decreases of the orbital periods possibly due to nonconservative mass transfer and angular momentum loss (see, e.g., Qian 2000a). To assess the significance of this effect on R CMa, we modified our fitting program by considering a quadratic term. The coefficient of this quadratic term was found to be $(-2.1 \pm 1.1) \times 10^{-11}$ day, which translates into a period decrease rate of $d P / d t=(-6.9 \pm 3.6) \times 10^{-9}$ day $\mathrm{yr}^{-1}$. This is a very slow rate compared to other Algol systems (see, e.g., Qian 2001) yet commensurate with the low activity level of R CMa, which is near the end of its mass transfer stage. Because of the poor significance (below $2 \sigma$ ) of the period decrease rate derived from the analysis, we decided to neglect the quadratic term and adopt a linear ephemeris.
TABLE 3

Astrometric AND LTT SOLUTIONS FOR THE Triple System R CMA

\begin{tabular}{|c|c|}
\hline Parameter & Value and Standard Error \\
\hline$\pi$ (mas) & $22.70 \pm 0.89$ \\
\hline$\mu_{\alpha} \cos \delta\left(\operatorname{mas} \mathrm{yr}^{-1}\right) \ldots \ldots \ldots \ldots$ & $168.1 \pm 0.7$ \\
\hline 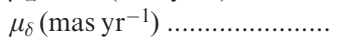 & $-137.1 \pm 1.2$ \\
\hline 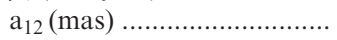 & $117.2 \pm 5.3$ \\
\hline$\Omega_{12}(\mathrm{deg})$ & $262.9 \pm 20.7$ \\
\hline$P_{12}(\mathrm{yr})$ & $92.8 \pm 1.3$ \\
\hline$e_{12}$ & $0.49 \pm 0.05$ \\
\hline$i_{12}(\mathrm{deg})$ & $91.7 \pm 4.7$ \\
\hline$\omega_{12}(\mathrm{deg}) \ldots \ldots \ldots \ldots \ldots \ldots \ldots$ & $10.5 \pm 4.3$ \\
\hline 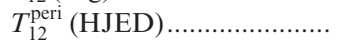 & $2449343 \pm 258$ \\
\hline LTT semiamplitude (s)....... & $2574 \pm 57$ \\
\hline$M_{12}\left(M_{\odot}\right)$ & $1.24 \pm 0.05^{\mathrm{a}}$ \\
\hline$M_{3}\left(M_{\odot}\right) \ldots \ldots \ldots \ldots \ldots \ldots$ & $0.34 \pm 0.02$ \\
\hline$a_{3}(\mathrm{AU})$ & $18.7 \pm 1.7$ \\
\hline$P_{\mathrm{EB}}$ (days) $\ldots \ldots \ldots \ldots \ldots \ldots \ldots$ & $1.13594197 \pm 0.00000010$ \\
\hline$T_{0 \mathrm{~EB}}(\mathrm{HJED}) \ldots \ldots \ldots \ldots \ldots$ & $2430436.5807 \pm 0.0006$ \\
\hline
\end{tabular}

a Adopted from Tomkin 1985.

It should be pointed out, however, that the astrometric and orbital parameters resulting from the fit with quadratic ephemeris are well within $1 \sigma$ of those listed in Table 3 .

Interestingly, a closer inspection of Figure 1 reveals small excursions of the data from the LTT fit. To investigate these, we computed the fit residuals that are shown in Figure 2 . The presence of low-amplitude cyclic deviations seems quite obvious in this plot. If these $(O-C)$ timing oscillations were caused by the perturbation of a fourth body in a circular orbit (R CMa D), its orbital period would be about 45 $\mathrm{yr}$, with an LTT semiamplitude of $275 \mathrm{~s}$, a minimum mass of $0.06 M_{\odot}$, and an orbital semimajor axis of about $14 \mathrm{AU}$. The orbit of the third body is highly eccentric so that it would be interior to that of $\mathrm{R} \mathrm{CMa} \mathrm{C}$ near its periastron $\left(r_{3 P}=9.5 \mathrm{AU}\right)$. The intersections of the two orbits would result in an apparently unstable configuration. Other possible explanations for the low-amplitude oscillations include abrupt period changes of the binary itself caused by variable angular momentum loss and magnetic coupling (see, e.g., Qian 2000b), a magnetic activity cycle of the secondary star (see, e.g., Applegate 1992), or simply a spurious effect caused by the inhomogeneity of the data set. Unfortunately, the available astrometric data are not sensitive enough to prove or refute the existence of a fourth body, and only new

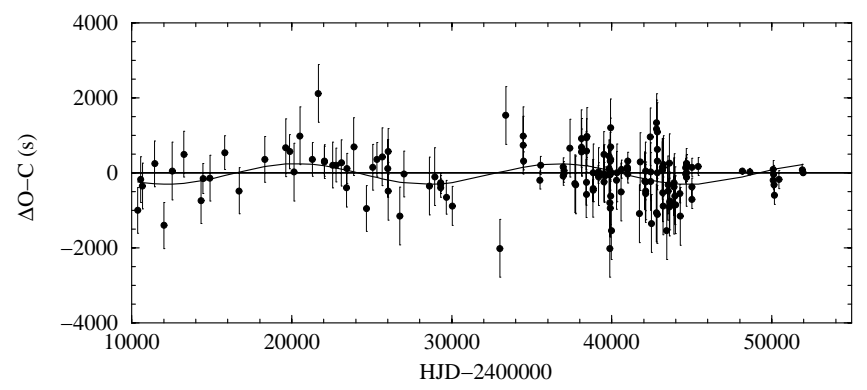

FIG. 2.-Residuals of the fit to the LTT orbit of the third body. The remaining oscillations have tentatively been modeled with an LTT perturbation caused by a fourth body in a circular orbit. See text for fit parameters. 
accurate photometric eclipse timing determinations or highaccuracy astrometry will provide the necessary evidence.

\section{DISCUSSION}

The orbital, astrometric, and physical properties presented in Table 3 are within $1 \sigma$ of the (less accurate) earlier estimates of Radhakrishnan et al. (1984), who based their analysis on eclipse timings up to 1982 . However, our study, in addition to extending the time baseline, has been able to determine the inclination of the third body's orbit by making use of the available high-precision astrometry (Hipparcos). Thus, R CMa joins Algol, the prototype of its class, in having the orbital properties of the third body determined from a combined analysis of the astrometry and LTT. The long period ( $\sim 93 \mathrm{yr})$ of $\mathrm{R} \mathrm{CMa} \mathrm{C}$ is the longest period detected and confirmed so far for an eclipsing binary. This is chiefly because of the large LTT present in R CMa (total amplitude of 86 minutes) and the existence of eclipse timings available for this star back to 1887 .

It is interesting to note that the inclination of $\mathrm{R} \mathrm{CMa} \mathrm{C}$ is found to be of $\approx 92^{\circ} \pm 5^{\circ}$ and thus compatible with an edgeon value of $90^{\circ}$. In this situation, mutual eclipses of the tertiary component and the close binary pair might occur. This tantalizing possibility is, however, very unlikely since eclipses are only possible within a very narrow window $(\sim 1 ! 2)$ about an inclination of $90^{\circ}$. If this were indeed the case, the transit of the tertiary component in front of the eclipsing pair should have occurred during mid 2001. Also interesting to note is the near coplanarity of the eclipsing system and its companion. Varricatt \& Ashok (1999) found an inclination for the eclipsing pair of $i_{\mathrm{EB}}=79^{\circ} .5$, which is equivalent to $i_{\mathrm{EB}}=100.5$ because of the degeneracy. Thus, the third body's orbit appears to be within only $8^{\circ}-13^{\circ}$ of the orbit of the eclipsing pair.

One question remains yet unaddressed, and this is the nature of the tertiary companion of $\mathrm{R} \mathrm{CMa}$. With a measured mass of $0.34 M_{\odot}$, one is tempted to classify R CMa C tentatively as a main-sequence M3-4 star (Delfosse et al. 2000). However, another attractive possible scenario is a white dwarf (WD) as tertiary component. There is no direct evidence for a WD companion to R CMa, but the mass of the third body is compatible with the low-end of the WD mass distribution found by Silvestri et al. (2001). The presence of a hot WD ( $\left.T_{\text {eff }}>10,000 \mathrm{~K}\right)$ is unlikely from IUE observations of $\mathrm{R} C \mathrm{CMa}$ in the UV region, where no hot source has been detected. Nonetheless, R CMa is an old disk population star so that a young WD is, in principle, not expected. If a WD is present, its original stellar mass would have to be greater than the mass of the initial primary (now secondary) of R CMa. From binary evolution theory, the best estimate of the initial mass of the original primary is about $1.4 M_{\odot}$ (Sarma, Vivekananda Rao, \& Abhyankar 1996). This indicates a pre-WD evolution time for the companion of around 2-3 Gyr. Cooling sequences for WDs (Serenelli et al. 2001) yield an effective temperature of $\sim 5400 \mathrm{~K}$ at an age of $\sim 3 \mathrm{Gyr}$, which is a reasonable estimate given the kinematic characteristics of R CMa. Should the tertiary component turn out to be a WD, such an old and low-mass object might be exceedingly interesting since it could belong to the controversial class of blue WDs that have been claimed to play an important role in explaining the dark matter content of the galactic halo (Hodgkin et al. 2000).
Since the predicted temperatures both in the WD and M star scenarios are fairly similar, only the very different expected luminosities can help identify the nature of the companion to R CMa. Thus, the measure of the magnitudes of the tertiary component through direct imaging would be a definitive proof. If we consider the $\mathrm{M}$ star scenario, the absolute magnitude of the tertiary component would be $M_{V} \approx 11 \mathrm{mag}$, which translates to $m_{V} \approx 14 \mathrm{mag}$ when using the parallax obtained in $\S 4$. This is about 8 mag fainter than $\mathrm{R}$ CMa itself. To give an example in the IR, the situation is significantly improved in the $K$ band, where the magnitude difference is reduced to $\Delta K \approx 4$ mag. The tertiary component would be even fainter in the WD scenario. Indeed, the absolute magnitude can be estimated as $M_{V} \approx 14 \mathrm{mag}$, which implies an apparent magnitude of $m_{V} \approx 17 \mathrm{mag}$. The difference with $\mathrm{R} \mathrm{CMa}$ is therefore $\Delta V \approx 11 \mathrm{mag}$. In the IR the situation does not improve significantly, with a large magnitude difference of $\Delta K \approx 10 \mathrm{mag}$.

These magnitudes and dynamic brightness ranges are challenging but yet attainable with state-of-the-art coronographs or Speckle spectrographs. Further complications arise from the current spatial location of the tertiary component near the conjunction of its orbit with the eclipsing pair. Figure 3 depicts the predicted orbits of both the eclipsing pair and the tertiary component on the plane of the sky. As can be seen, the separation between the eclipsing system and $\mathrm{R} \mathrm{CMa} \mathrm{C}$ is only 27 mas as of 2002, which makes direct imaging very difficult. On an optimistic note, the situation will slowly improve in the future until a maximum separation of $\approx 0$ "! 8 is reached around year 2037 .

Claims of third-body detections through the analysis of $(\mathrm{O}-\mathrm{C})$ residuals have sometimes been challenged. Spurious period changes caused by magnetic activity cycles, variable angular momentum loss, magnetic coupling, or other effects have been argued to explain modulations in the $(O-C)$ residuals found in a number of eclipsing binary stars. Interestingly, R CMa would be a prime candidate for such spurious period changes because of its interactive nature.

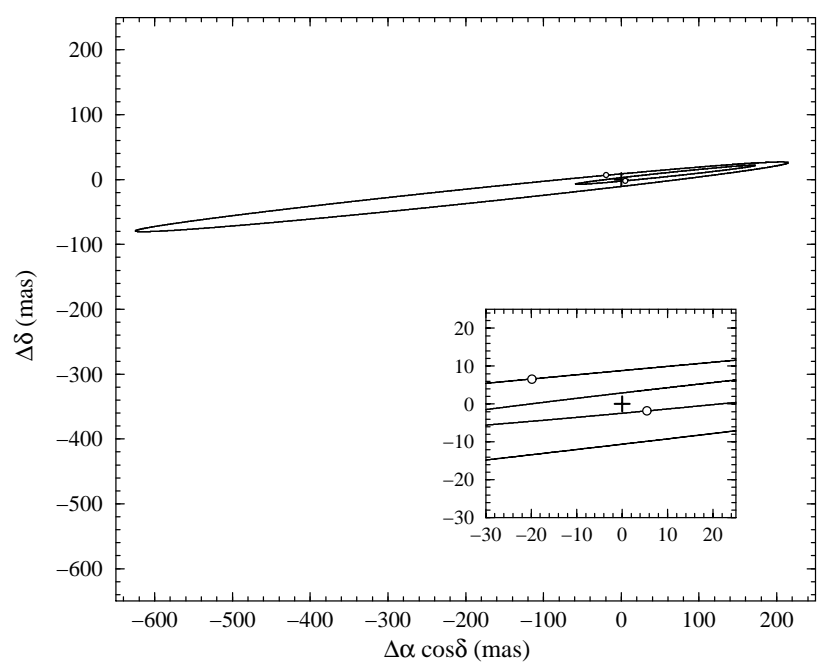

FIG. 3.- Scale projection on the plane of the sky of the orbits of the eclipsing pair of R CMa (small ellipse) and the tertiary component (large ellipse). The barycenter of the triple system is marked with a plus sign, and the positions of the stars in 2002 are represented as open circles. The inset shows a blowup of the region surrounding the barycenter. The orbital properties and the sky projection were derived from the simultaneous analysis of eclipse timing residuals and Hipparcos astrometry (see $\S 4$ ). 
However, with over one period cycle in the LTT curve currently covered and, more importantly, with direct evidence from Hipparcos astrometry, the case for a tertiary companion to $\mathrm{R} \mathrm{CMa}$ is now ironclad. What only remains to be clarified at this point is whether this third star is an M dwarf or a WD. Also, the nature of the lower amplitude $\sim 45 \mathrm{yr}$ variation needs to be further explored with continued observations.

\section{CONCLUSIONS}

This paper presents a combined analysis of short-term accurate astrometry and long-term timing residuals applied to the eclipsing binary R CMa. The study yields the complete orbital and physical properties of the tertiary component. A determination of the mass of the third body is possible because the masses of the eclipsing binary components themselves are well known from light and radial velocity curve analyses.

The example discussed here illustrated the capabilities of a method that will reach its full potential with the upcoming high-accuracy astrometric missions. The improvements in precision of the future astrometric measurements are due to an increase of up to a thousandfold relative to Hipparcos, and the quality of the photometry (and thus the eclipse timings) will also improve. More quantitatively, timings with accuracies of $\sim 10 \mathrm{~s}$ are now possible for select eclipsing binaries with sharp eclipses. The detection of large planets $\left(\sim 10 M_{\mathrm{J}}\right)$ in long-period orbits $(\sim 10-20 \mathrm{yr})$ around eclipsing binaries will be therefore a relatively easy task. The shortterm astrometry will confirm the detections and yield the complete orbital solution (most significantly the inclination) and thus the actual mass of the orbiting body.

One of the unexpected outcomes of the Hipparcos mission has been that a primarily astrometric satellite can also provide valuable new results from its photometric measurements alone (numerous new variables, HD 209458 planetary transits, etc.). The data analysis of the next generations of astrometric satellites will surely benefit from a simultaneous analysis of the astrometric and photometric data. Astrometric missions such as GAIA will likely detect one million new eclipsing binaries (a smaller number is expected for $F A M E$ ). About $1 \%$ of the eclipsing binaries observed by Hipparcos has a 0.0001 day precision in the reference epoch, which is enough to detect the LTT effect that would arise from a $10 M_{\mathrm{J}}$ third body with an $11 \mathrm{yr}$ period. If we assume the same ratio for GAIA, hundreds to thousands of third bodies would be detected. Although GAIA astrometry alone will be able to give the orbits for the closest stars, the orbit for more distant stars will depend on the availability of ground-based light curves to define the reference epoch.

This method of combining LTT analysis and astrometry complements very well the ongoing spectroscopic searches. The LTT analysis favors the detection of long-period third bodies around eclipsing binaries because the amplitude of the time delay due to the LTT effect is proportional to $P_{12}^{2 / 3}$, while the spectroscopic semiamplitude is proportional to $P_{12}^{-1 / 3}$. When the samples of spectroscopic and LTT systems are sufficiently large, we will have a complete picture of the distribution of bodies in a stellar system, and a realistic test of planet formation theories will be possible.

Finally, the LTT analysis method does not have to be necessarily applied to eclipsing binaries. In essence, the method is based on having a "beacon in orbit," which, in the case of eclipsing binaries, are the mideclipse times. However, any strictly periodic event that can be predicted with good accuracy could be potentially useful to detect stellar or substellar companions. This includes, for example, pulsating stars. More interestingly, transiting planets are also prime candidates for LTT studies. In this case, not only could further orbiting planets be discovered, but also good chances for detecting moons around the transiting planet exist.

S. E. Urban (US Naval Observatory), who provided the older epoch astrometric data of R CMa is warmly thanked. The APT observations were acquired and reduced using programs developed by G. P. McCook, who is gratefully acknowledged. Also, we thank astronomy students J. J. Bochanski and D. Stack for help with data preparation for this work. The referee, O. Demircan, is thanked for a number of important comments and suggestions that led to the improvement of the paper. I. R. thanks the Catalan Regional Government (CIRIT) for financial support through a Fulbright fellowship. This research was supported by NSF/RUI grants AST 93-15365, AST 95-28506, and AST 00-71260.

\section{REFERENCES}

Applegate, J. H. 1992, ApJ, 385, 621

Arenou, F. 2001, in École de Goutelas 23, ed. D. Egret, J.-L. Halbwachs, \& J.-M. Hameury (Paris: Société Française d'Astronomie et d'Astrophysique), 127

Bachmann, P. J., \& Hershey, J. L. 1975, AJ, 80, 836

Bastian, U. 2000, Inf. Bull. Variable Stars, 4822,

Bystrov, N. F., Polojentsev, D. D., Potter, H. I., Yagudin, L. I., Zalles, R. F., \& Zelaya, J. A. 1991, in IAU Colloq. 127, Reference Systems, ed. J. A. Hughes, C. A. Smith, \& G. H. Kaplan (Washington: USNO), 216

Deeg, H. J., Doyle, L. R., Kozhevnikov, V. P., Blue, J. E., Martín, E. L., \& Schneider, J. 2000, A\&A, 358, L5

Delfosse, X., Forveille, T., Ségransan, D., Beuzit, J.-L., Udry, S., Perrier, C., \& Mayor, M. 2000, A\&A, 364, 217

Demircan, O. 2000, in Variable Stars as Essential Astrophysical Tools, ed. C. İbanoĝlu (NATO ASI Ser. C, 544; Dordrecht: Kluwer), 615

Dugan, R. S., \& Wright, F. W. 1939, Princeton Contrib., 19, 34

Edalati, M. T., Khalesse, B., \& Riazi, N. 1989, Ap\&SS, 151, 1

Fabricius, C. 1993, Bull. Inf. Cen. Donnes Stellaires, 42, 5

Guinan, E. F. 1977, AJ, 82, 51

Guinan, E. F., \& Ianna, P. A. 1983, AJ, 88, 126

Guinan, E. F., \& Ribas, I. 2001, ApJ, 546, L43
Halbwachs, J. L., Arenou, F., Mayor, M., Udry, S., \& Queloz, D. 2000, A\&A, 355, 581

Hodgkin, S. T., Oppenheimer, B. R., Hambly, N. C., Jameson, R. F., Smartt, S. J., \& Steele, I. A. 2000, Nature, 403, 57

Irwin, J. B. 1952, ApJ, 116, 211

Kitamura, M. 1969, Ap\&SS, 3, 163

Knipe, G. F. G. 1963, Rep. Obs Johannesburg Circ., 7, 122, 21

Koch, R. H. 1960, AJ, 65, 326

McArthur, B., Jefferys, W., \& McCartney, J. 1994, BAAS, 184, 28.04

Mkrtichian, D. E., \& Gamarova, A. Yu. 2000, Inf. Bull. Variable Stars, 4836, 1

Perryman, M. A. C. 1997, The Hipparcos and Tycho Catalogues (ESA SP-1200; Noordwijk: ESA)

Qian, S. 2000a, AJ, 119, 901

.2000b, AJ, 119, 3064 2001, AJ, 121, 1614

Radhakrishnan, K. R., Sarma, M. B. K., \& Abhyankar, K. D. 1984 Ap\&SS, 99, 229

Robinson, L. J. 1967, Inf. Bull. Variable Stars, 180, 1

Sarma, M. B. K., Vivekananda Rao, P., \& Abhyankar, K. D. 1996, ApJ, 458,371 
Sawyer, E. F. 1887, AJ, 7, 119

Serenelli, A. M., Althaus, L. G., Rohrmann, R. D., \& Benvenuto, O. G. 2001, MNRAS, 325, 607

Silvestri, N. M., Oswalt, T. D., Wood, M. A., Smith, J. A., Reid, I. N., \& Sion, E. M. 2001, AJ, 121, 503

Tomkin, J. 1985, ApJ, 297, 250

Urban, S. E., Corbin, T. E., Wycoff, G. L., Martin, J. C., Jackson, E. S., Zacharias, M. I., \& Hall, D. M. 1998, AJ, 115, 1212
Varricatt, W. P., \& Ashok, N. M. 1999, AJ, 117, 2980

Wilson, R. E., \& Devinney, E. J. 1971, ApJ, 166, 605

Wolszczan, A., \& Frail, D. A. 1992, Nature, 355, 145

Wood, F. B. 1946, Princeton Contrib., 21, 31

Zacharias, N., de Vegt, C., Nicholson, W., \& Penston, M. J. 1992, A\&A, 254,397 